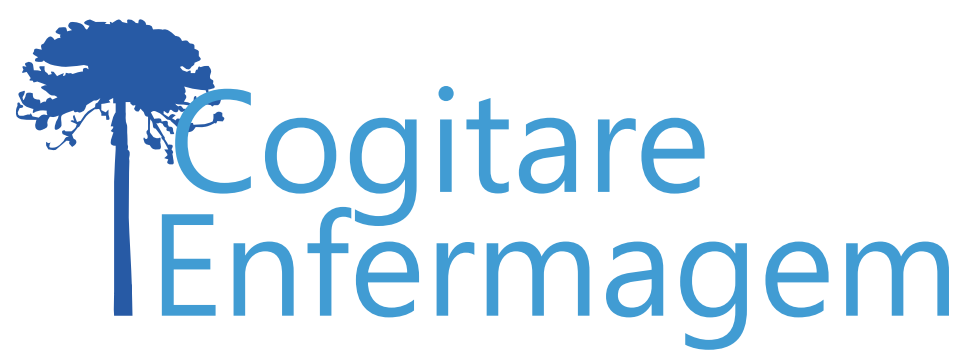

\title{
CUSTO DIRETO DA INSERÇÃO DE CATETER VENOSO CENTRAL PARA REALIZAÇÃO DE HEMODIÁLISE CONVENCIONAL
}

\author{
Bruno Leite de Azevedo Carneiro ${ }^{1}$ (1) \\ Ana Cláudia Tavares de Melo ${ }^{1}$ (i) \\ Antônio Fernandes Costa Lima ${ }^{1}$ [1]
}

\begin{abstract}
RESUMO
Objetivo: identificar o custo direto médio da inserção de cateter venoso central de longa permanência em pacientes submetidos à hemodiálise convencional em um hospital público de ensino e pesquisa.

Método: pesquisa quantitativa, exploratório-descritiva, realizada em um Centro de Diálise, em São Paulo, Brasil entre novembro e dezembro/2019. Calculou-se o custo direto médio multiplicandose o tempo despendido por profissionais de saúde pelo custo unitário da mão de obra direta, somando-se aos custos de insumos.

Resultados: o custo direto médio total correspondeu a US\$134,56 (DP $\pm 3,65$ ), sendo US $\$ 107,01$ $(\mathrm{DP} \pm 0,23)$ relativos ao custo com material, US $\$ 22,10$ (DP $\pm 3,63)$ com a mão de obra direta da equipe de inserção do cateter, US $\$ 4,65$ (DP $\pm 0,00)$ com custo de medicamentos e US $\$ 0,80(\mathrm{DP} \pm 0,15)$ com custo de soluções.

Conclusão: constatou-se o impacto dos custos com recursos materiais e a imprescindibilidade da sua alocação racional, especialmente nos hospitais públicos de ensino e pesquisa, que possuem recursos financeiros limitados.
\end{abstract}

DESCRITORES: Insuficiência Renal Crônica; Unidades Hospitalares de Hemodiálise; Enfermagem em Nefrologia; Cateterismo Venoso Central; Custos e Análise de Custo.

\section{COSTO DIRECTO DE LA INSERCIÓN DE CATÉTER VENOSO CENTRAL PARA REALIZACIÓN DE LA HEMODIÁLISIS CONVENCIONAL}

\section{RESUMEN:}

Objetivo: identificar el costo directo de la inserción de catéteres venosos centrales de larga duración en pacientes sometidos a hemodiálisis convencional en un hospital público de enseñanza e investigación. Método: investigación cuantitativa exploratoria-descriptiva realizada en un Centro de Diálisis de São Paulo, Brasil, entre noviembre y diciembre/2019. El costo directo medio se calculó multiplicando el tiempo empleado por los profesionales de la salud por el costo unitario de la mano de obra directa, sumando los costos de los insumos. Resultados: el costo directo medio total fue de 134,56 dólares (SD $\pm 3,65)$, de los cuales 107,01 dólares $(S D \pm 0,23)$ fueron costos de material, 22,10 dólares $(S D \pm 3,63)$ fueron costos de mano de obra directa del equipo de inserción de catéteres, 4,65 dólares $(S D \pm 0,00)$ fueron costos de medicación y 0,80 dólares $(S D \pm 0,15)$ fueron costos de solución. Conclusión: se ha constatado el impacto de los costos de los recursos materiales y la importancia de su asociación racional, especialmente en los hospitales públicos de enseñanza e investigación, que cuentan con recursos financieros limitados.

DESCRIPTORES: Insuficiencia Renal Crónica; Unidades de Hemodiálisis en Hospital; Enfermería en Nefrología; Cateterismo Venoso Central; Costos y Análisis de Costo. 
No Brasil, a elevação na prevalência de portadores de doença renal crônica (DRC) tem se constituído em importante problema de saúde pública(1), semelhantemente ao que ocorre em outros países ${ }^{(2)}$. A incidência de DRC tem aumentado devido ao crescimento no quantitativo de indivíduos diabéticos, hipertensos e do envelhecimento populacional(2).

A DRC ocorre silenciosamente e os portadores podem descobrir sua existência somente em estágios mais avançados, que demandam a adoção de uma modalidade de Terapia Renal Substitutiva (TRS). O tratamento indicado varia de acordo com o estágio da DRC, podendo ser conservador, com o uso de dieta e medicamentos; dialítico, por meio da hemodiálise (HD) ou diálise peritoneal (intermitente, ambulatorial contínua, automatizada); ou pela realização de transplante renal(3).

A hemodiálise convencional (HDC) tem sido tradicionalmente adotada há décadas, especialmente para os estágios mais avançados da DRC (4). Nos Centros de Diálise (CDs), a assistência prestada aos pacientes em programa de HDC abrange procedimentos e ações complexos, necessitando de recursos que contemplem as disposições legais vigentes $\mathbf{s}^{(5-6)}$. Requer a utilização de soluções, materiais e equipamentos específicos, disponibilidade de equipe especializada durante a realização de todo o tratamento, estrutura física apropriada e um eficiente acesso vascular (AV) à circulação sanguínea ${ }^{(7)}$.

O êxito da HDC está diretamente relacionado à adequação do $A V^{(8)}$, que pode ser obtido por meio confecção de Fístula Arteriovenosa (FAV) autóloga, enxerto arteriovenoso protético ou por inserção de cateter venoso central $(\mathrm{CVC})^{(9)}$. Ressalta-se que o AV precisa apresentar facilidade de utilização, bom fluxo sanguíneo, baixa resistência no retorno venoso, durabilidade garantida e baixa probabilidade de acidentes hemorrágicos, coagulação e infecção(8).

Frente à ocorrência de complicações que comprometam/impossibilitem o uso do AV e, consequentemente, inviabilizem a realização da sessão de HDC, torna-se necessária a adoção de alguma intervenção imediata, como exemplo, a inserção ou reinserção de CVC, gerando custos que precisam ser identificados e devidamente gerenciados.

Na dimensão gerencial, a apuração dos custos dos serviços de saúde é imprescindível para direcionar a alocação racional dos insumos consumidos ${ }^{(10)}$. Entretanto, estudos sobre os custos relativos aos procedimentos para o manejo das complicações associadas ao AV para HDC ainda são escassos, principalmente no contexto nacional, como verificase em relação ao procedimento de inserção de CVC de longa permanência. Pesquisas desta natureza podem gerar novos conhecimentos a respeito dos custos associados a intervenções de resgate para viabilizar a HDC e auxiliar na identificação, monitoramento e gerenciamento dos custos na prática assistencial dos CDs.

Nesta perspectiva, realizou-se este estudo objetivando identificar o custo direto médio da inserção de cateter venoso central de longa permanência em pacientes submetidos à hemodiálise convencional em um hospital público de ensino e pesquisa.

\section{MÉTODO}

Pesquisa quantitativa, exploratório-descritiva, na modalidade estudo de caso único, realizada no CD de um Hospital Público de Ensino e Pesquisa (HPEP), que é uma entidade autárquica de Regime Especial, associada a uma Universidade Pública mantida pelo Estado 
de São Paulo e ligada à Secretaria de Estado de Desenvolvimento Econômico, Ciências e Tecnologia, por intermédio de uma de suas Unidades de Ensino.

$\mathrm{O} C D$ cumpre plenamente as disposições legais relativas à adequada estrutura tecnológica e aos recursos humanos quanti-qualitativos necessários ao atendimento das demandas de cuidados dos portadores de DRC em programa de HDC (9-10). Dispõe de 24 máquinas para HDC e atende 118 pacientes alocados em três turnos de segunda-feira a sábado. A equipe é composta por médicos residentes e preceptores em Nefrologia, enfermeiros e técnicos/auxiliares de enfermagem (TE/AE), nutricionista, psicóloga e assistente social.

No HPEP, é padronizado que a inserção de CVC de longa permanência, como via de acesso para viabilização da HDC, seja realizada por um médico residente, sob a supervisão de um médico preceptor, ambos da área de Nefrologia, e com auxílio de um TE/AE do CD.

Tendo em vista que não há variação do quantitativo de recursos humanos (médico residente, médico preceptor e TE/AE) envolvidos no procedimento de inserção de CVC de longa permanência e pouca variação no quantitativo de insumos consumidos (materiais/ medicamentos/soluções), um profissional estatístico estabeleceu que apenas algumas observações seriam suficientes para a obtenção do custo direto médio (CDM). Assim, a amostra de conveniência correspondeu às oportunidades de observação direta, não participante do procedimento em estudo, realizado no HPEP nos períodos da manhã e tarde, entre novembro e dezembro de 2019.

Foram realizadas observações não participantes para documentação do quantitativo e da categoria de profissionais de saúde envolvidos na inserção/auxílio da inserção de CVC de longa permanência; do tempo (cronometrado) por eles despendido; dos materiais/ medicamentos/soluções e quantidades utilizadas no procedimento. Para a obtenção do custo direto médio deste procedimento [ $\overline{C\left(P_{2}\right)}$ ], realizou-se um estudo de micro custeio cujo referencial consistirá na apuração dos custos diretos.

Custos diretos são aqueles que podem ser identificados e claramente quantificados; referem-se a um dispêndio monetário aplicado na produção de um produto ou de um serviço no qual é possível a identificação com o produto ou departamento ${ }^{(11)}$. Nas organizações hospitalares, os custos diretos são compostos pela mão de obra direta (MOD), insumos e equipamentos utilizados no processo assistencial|(12).

A MOD refere-se ao pessoal que trabalha diretamente sobre um produto/serviço prestado, desde que seja possível mensurar o tempo despendido e identificar quem executou o trabalho. Compõe-se dos salários, encargos sociais, provisões para férias e $13^{\circ}$ salário(11). O custo unitário da MOD foi calculado a partir dos salários médios de TE/AE do $C D$ e valores das bolsas de médicos residentes e médicos preceptores da Nefrologia fornecidos pelo Serviço de Recursos Humanos do HPEP.

Os preços dos materiais e soluções/medicamentos consumidos na inserção do CVC de longa permanência foram obtidos junto ao Departamento de Compras/Almoxarifado do HPEP.

Para a apuração do $\overline{C\left(P_{1}\right)}$ foram identificadas a quantidade média dos materiais $\left[\overline{q m_{k}}\right] ;$ o preço unitário médio de cada material $\left[\overline{\mathrm{Pmu}_{k}}\right]$; a quantidade média das soluções/medicamentos [ $\overline{q s_{k}}$ ]; o preço unitário médio de cada solução/medicamento [ $\bar{P} u_{k}$ ] ; o tempo médio de dedicação de cada categoria profissional [ ${ }_{c}$ ] e a massa salarial unitária média da MOD de cada categoria profissional [ $\overline{S u_{c}}$ ] obtendo-se a equação: $\overline{C\left(P_{2}\right)}=\sum_{k=1}^{n}\left(\overline{q_{k}} \cdot \overline{P u_{k}}\right)+\sum_{k=1}^{n}\left(\overline{q s_{k}} \cdot \overline{P s u_{k}}\right)+\sum_{c=1}^{n}\left(\overline{t_{c}} \cdot \overline{S u_{c}}\right)^{(10)}$.

Para calcular o CDM da inserção de CVC de longa permanência, utilizou-se originalmente a moeda brasileira real $(R \$)$, que foi convertida posteriormente para o dólar americano (US\$) considerando a taxa de conversão de US\$1,00/R $\$ 5,46$, com base na cotação de 01/02/2021, fornecida pelo Banco Central do Brasil. 
O estudo foi aprovado pelos Comitês de Ética e Pesquisa da Instituição proponente e do HPEP por meio dos pareceres consubstanciados números: 3.324.582, em 14/05/2019 e 3.373.665, em 06/06/2019, respectivamente.

\section{RESULTADOS}

Entre novembro e dezembro de 2019, foram realizadas 11 observações de inserção de CVC de longa permanência. Os salários médios recebidos pelos profissionais integrantes do procedimento corresponderam a: médico preceptor (bolsa de preceptoria e vale alimentação) - US $\$ 1056,49 / 160$ horas, US $\$ 6,60 /$ hora e US $\$ 0,11 /$ minuto; médico residente (bolsa de residência) - US\$609,97/240 horas, US\$2,54/hora e US\$0,04/minuto; e TE/AE (massa salarial: salários base, benefícios, gratificações e encargos sociais) - US\$873,02/120 horas, US\$7,27/hora e US\$0,12/minuto.

No que diz respeito aos custos dos insumos utilizados, verifica-se no Quadro 1 o predomínio dos itens Kit CDL-LP carbothano 14,5fr, $28 \mathrm{~cm}$ para HD (unidade) - US\$103,48; Heparina sódica $5000 \mathrm{UI} / \mathrm{ml}$ solução injetável (IV) (frasco $5 \mathrm{ml}$ ) - US $\$ 2,66$; Cefazolina pó solução injetável (frasco 1 grama) - US\$0,92; Lidocaína (cloridrato) 2\% solução injetável (frasco 20ml) - US\$0,47 e Cloreto de sódio 0,9\% solução injetável (bolsa 100ml) - US\$0,23.

Quadro 1 - Distribuição dos custos dos materiais e soluções/medicamentos utilizados no procedimento de inserção de CVC de longa permanência. São Paulo, SP, Brasil, 2020 (continua)

\begin{tabular}{|l|c|}
\hline Material e solução/medicamento (apresentação) & $\begin{array}{c}\text { Custo/custo estimado* } \\
\text { em US\$ }\end{array}$ \\
\hline $\begin{array}{l}\text { Agulha hipodérmica descartável 30x7, com dispositivo de segurança } \\
\text { (unidade) }\end{array}$ & 0,03 \\
\hline Agulha hipodérmica descartável com ponta romba (unidade) & 0,01 \\
\hline Bola de algodão (unidade) & 0,004 \\
\hline $\begin{array}{l}\text { Cateter para oxigênio descartável com prolongamento nasal tipo óculos } \\
\text { (unidade) }\end{array}$ & 0,06 \\
\hline Compressa gaze estéril 7,5x7,5cm sem filamento radiopaco (pacote) & 0,15 \\
\hline $\begin{array}{l}\text { Equipo para administração de solução parenteral macrogotas com injetor } \\
\text { lateral de infusão 150cm (unidade) }\end{array}$ & 0,25 \\
\hline Escova descartável com clorexidina degermante 2\% (unidade) & 0,19 \\
\hline $\begin{array}{l}\text { Fio de nylon 3/0, com 1 agulha 3/8, círculo triangular 3/0 cm diâmetro, 450 } \\
\text { cm comprimento (envelope) }\end{array}$ & \begin{tabular}{c} 
\\
\hline Fita adesiva cirúrgica de rayon (rolo 10cmx450cm)
\end{tabular} \\
\hline Kit CDL-LP carbothano 14,5fr, 28 cm para HD (unidade) & 2,49 (rolo 10cmx450cm) \\
\hline Lâmina descartável para bisturi (par) & 0,8 (15cm/procedimento) \\
\hline Luva para procedimento cirúrgico estéril (par) & 0,37 \\
\hline Luva sintética (nitrílica), para procedimento, não cirúrgico, não estéril (par) & 0,11 \\
\hline Máscara cirúrgica descartável (unidade) & 0,05 \\
\hline Seringa descartável 10ml (unidade) & 0,02 \\
\hline
\end{tabular}




\begin{tabular}{|l|c|}
\hline Seringa descartável 20ml (unidade) & 0,06 \\
\hline Seringa descartável 3ml (unidade) & 0,02 \\
\hline Seringa descartável 5ml (unidade) & 0,02 \\
\hline Touca cirúrgica descartável (unidade) & 0,01 \\
\hline Cloreto de sódio 0,9\% 10ml (unidade) & 0,02 \\
\hline Cloreto de sódio 0,9\% solução injetável (bolsa 100ml) & 0,23 \\
\hline Clorexidina alcoólica 0,5\% (frasco 100ml) & 0,31 (frasco 100ml) \\
\hline Cefazolina pó solução injetável (frasco 1 grama) & 0,06 (20ml/procedimento) \\
\hline Fentanila (citrato) 0,05 mg/ml solução injetável (ampola 5 ml) & 0,92 \\
\hline Heparina (sódica) 5000 Ul/ml solução injetável (IV) (frasco 5ml) & 0,4 \\
\hline Lidocaína (cloridrato) 2\% solução injetável (frasco 20ml) & 2,66 \\
\hline Midazolam 5mg/ml solução injetável (ampola 3ml) & 0,47 \\
\hline
\end{tabular}

Fonte: Autores (2020)

O tempo de duração da realização dos 11 procedimentos de "inserção de CVC de longa permanência para HD" variou entre 63,00 e 111,00 minutos, com média de 81,55 (DP $\pm 13,39)$ e mediana de 79,00 minutos.

Constata-se, na Tabela 1, que o CDM total correspondeu a US\$134,56 $(\mathrm{DP} \pm 3,65)$ sendo o custo com material (US $\$ 107,01-\mathrm{DP} \pm 0,23)$ a variável de maior impacto $(79,52 \%$ do CDM total) com destaque ao Kit CDL-LP carbothano para HD item de maior custo unitário (US\$103,48). Ressalta-se também a representatividade do custo com MOD da equipe de inserção do CVC de longa permanência (US\$22,10 - DP $\pm 3,63$ ) na composição do CDM total $(16,42 \%)$.

Tabela 1 - Distribuição das observações do procedimento "inserção de CVC de longa permanência para HD" considerando os custos das variáveis em análise. São Paulo, SP, Brasil, 2020

\begin{tabular}{lccccc} 
Observações & $\mathbf{n}$ & $\begin{array}{c}\text { Média } \\
\text { US\$ }\end{array}$ & $\begin{array}{c}\text { DP士 } \\
\text { US\$ }\end{array}$ & $\begin{array}{c}\text { Mediana } \\
\text { US\$ }\end{array}$ & $\begin{array}{c}\text { Valores mínimos } \\
\text { e máximos US\$ }\end{array}$ \\
\hline Custo com MOD de TE/AE* & 11 & 9,56 & 1,57 & 9,26 & $7,38-13,01$ \\
\hline Custo com MOD de médico residente** & 11 & 3,58 & 0,59 & 3,47 & $2,77-4,88$ \\
\hline Custo com MOD de médico preceptor ${ }^{\star \star *}$ & 11 & 8,96 & 1,47 & 8,68 & $6,92-12,20$ \\
\hline $\begin{array}{l}\text { Custo com MOD da equipe de inserção do } \\
\text { CVC de longa permanência }\end{array}$ & 11 & 22,1 & 3,63 & 21,27 & $17,08-30,09$ \\
\hline Custo com materiais & 11 & 107,01 & 0,23 & 107,09 & $106,53-107,27$ \\
\hline Custo com medicamentos & 11 & 4,65 & 0 & 4,65 & $4,65-4,65$ \\
\hline Custo com soluções & 11 & 0,8 & 0,15 & 0,73 & $0,62-1,06$ \\
\hline CDM Total & 11 & 134,56 & 3,65 & 133,81 & $130,07-143,00$
\end{tabular}

*MOB TE/AE: US\$0,12/minuto; ** MOB médico residente: US\$0,04/minuto; *** MOB médico preceptor US\$0,11/minuto.

Fonte: Autores (2020) 
Para a obtenção de melhores resultados em relação ao AV para HD, precisam ser considerados os aspectos físicos e o estilo de vida do paciente, as metas, as circunstâncias da criação do AV, a infraestrutura e a cultura/filosofia quanto ao AV vigentes nos CDs ${ }^{(13)}$. Nesta ótica, a confecção e a manutenção do AV, visando à eficiência da HD e qualidade de vida do paciente, impactam os custos atribuídos à DRC, contribuindo para a sobrecarga financeira associada a esta modalidade de TRS ${ }^{(14)}$.

As 11 passagens de CVC de longa permanência observadas neste estudo foram executadas por médicos residentes, sob a supervisão de um médico preceptor, ambos da área de nefrologia, e auxílio de um TE/AE do CD, conforme padronizado no HPEP. Não foram verificadas variações no quantitativo e categorias de profissionais envolvidos, bem como na quantidade dos materiais utilizados; observou-se apenas pouca variação no tempo despendido. Tal achado mostra que, por se tratar de um HPEP, o custo com a equipe de inserção do CVC de longa permanência é condizente com o esperado e evidencia a alocação racional dos recursos humanos e materiais envolvidos, coerente com os princípios, valores e filosofia de instituições desta natureza.

A padronização de procedimentos favorece a implementação de práticas com maior índice de segurança, garantindo confiabilidade à assistência prestada, possibilitando a organização dos processos de trabalho, o gerenciamento dos cuidados prestados e conferindo suporte aos profissionais de saúde ao criar ambiente propício para o ensino(15). Destaca-se a contribuição imprescindível da padronização dos insumos a serem utilizados nos procedimentos, de forma a evitar a ocorrência de desperdícios que comprometem a distribuição de recursos limitados e escassos.

Este fato pode ser observado no Canadá que, desde 2011, passou pela implementação de reformas de financiamento hospitalar com o objetivo de incentivar a padronização de procedimentos, definindo-se um preço fixo para cada procedimento hospitalar, com base em evidências científicas e estudos de custeio, melhorando a qualidade da assistência e reduzindo/minimizando os custos do sistema de saúde ${ }^{(16)}$.

Tendo em vista a alocação racional de recursos, de forma a incrementar o processo de cuidar, há que se considerar que o presente estudo foi realizado em um HPEP, cuja definição explicita tratar-se de instituições que oferecem assistência médica altamente complexa e que realizam, além de atividades de ensino, pesquisa e extensão de serviços à comunidade, o treinamento dos profissionais de saúde nos níveis primário, secundário e terciário(17).

Assim, a execução do procedimento "inserção de CVC de longa permanência", pelo médico residente, vai ao encontro da missão de um HPEP, sendo considerada uma estratégia adequada na medida em que contribui para a redução das demandas por serviços de saúde e para o aumento da qualidade assistencial prestada, ao modelar cientificamente as atividades preconizadas por meio da associação entre serviço, ensino e pesquisa ${ }^{(18)}$.

Em um cenário de gastos hospitalares crescentes, atrelados à escassez de recursos financeiros, a relevância do controle de custos tem se tornado cada vez mais evidente ${ }^{(19)}$. No Brasil, os recursos financeiros, materiais e humanos são escassos e limitados, requerendo, nos últimos anos, várias iniciativas para integrar evidências científicas no processo coletivo de tomada de decisão(20).

O desenvolvimento de pesquisas de custo para avaliações de procedimentos de saúde é crucial devido à mudança do perfil assistencial, forte inclusão tecnológica e presença de doenças emergentes ${ }^{(21)}$. Portanto, a geração de informações sobre custos é essencial para o gerenciamento dos recursos em saúde, apoiando os processos decisórios institucionais, seu monitoramento e avaliação. Porém, ainda há escassez de base de 
informações de custos no Brasil, limitando-se apenas a algumas instituições ${ }^{(21)}$. Por sua vez, a dinâmica da inovação tecnológica tem sido reconhecida como uma das razões para o aumento dos gastos em saúde e é um dos maiores desafios a ser enfrentado no Sistema Único de Saúde (SUS) ${ }^{(22)}$.

Compreendendo a relevância do monitoramento econômico adequado, o SUS criou o Sistema de Gerenciamento da Tabela de Procedimentos, Medicamentos, Órteses, Próteses e Materiais Especiais (SIGTAP), ferramenta gerencial que permite acompanhar sistematicamente, por meio de série histórica, as mudanças ocorridas em cada competência incluída no sistema. Possibilita a geração de diversos relatórios, fornecendo notas técnicas mensais, registrando as principais modificações efetuadas na Tabela de Procedimentos ${ }^{(23)}$. Entretanto, os valores praticados por essa Tabela, mesmo após reajustes (que não ocorrem frequentemente), não acompanham a inflação intrínseca do setor saúde, cujos preços crescem mais do que a economia em geral.

Comparando-se os valores repassados pelo SUS ao HPEP, constantes do SIGTAP(24), para o "cateter de longa permanência para hemodiálise" (R\$ 482,34) e "implante de cateter de longa permanência para hemodiálise" (R\$ 200,00) com os custos mensurados neste estudo, Kit CDL-LP carbothano para HD (R\$565,00 - US\$103,48) e passagem do CVC de longa permanência para hemodiálise (R\$169,72 - US\$31,09), verifica-se a diferença de menos $\mathrm{R} \$ 82,66$ (US $\$ 15,14$ ) e mais $\mathrm{R} \$ 30,28$ (US $\$ 5,54$ ) respectivamente, totalizando um déficit de cobertura de R\$52,38 (US\$9,60) por passagem de CVC de longa permanência. Diante desse resultado, concorda-se com estudo ${ }^{(25)}$ que explicita que o conhecimento do déficit de cobertura tem potencial para fundamentar a negociação entre os HPEP, contratualizados junto ao SUS, e o gestor estadual ou municipal para ajustes do repasse financeiro.

Neste estudo, foi possível observar que o Kit CDL-LP carbothano para HD foi o item de maior custo unitário, destacando-se em relação ao custo de material utilizado para a execução deste procedimento. $O$ uso do CDL de longa permanência é considerado pertinente tendo em vista que, sob a singular perspectiva que os nefrologistas têm em relação a diálise, o implante deste tipo de cateter tem demonstrado resultados eficazes e seguros para o paciente submetido a HDC (26).

Apesar do custo com materiais ter sido o mais expressivo na composição do CDM total da inserção de passagem de CVC de longa permanência para HD, à semelhança dos resultados de outros estudos sobre custeio de procedimentos hospitalares ${ }^{(27-29)}$, reitera-se a contribuição inegável, conforme anteriormente mencionado, da padronização dos insumos a serem utilizados no procedimento. Salienta-se ainda que, pela representatividade dos custos com recursos materiais, as instituições hospitalares, públicas e privadas, precisam realizar investimentos no tocante ao gerenciamento adequado desses recursos, aspecto básico que oferece possibilidade de redução de custos e de prejuízos ao orçamento de saúde nacional(30).

Indica-se, como limitação deste estudo, a opção pelo custeio direto do procedimento em questão, tendo em vista a impossibilidade de obtenção de informações, junto ao HPEP, referentes aos custos indiretos que permitiriam a aferição do custo total.

O CDM total do procedimento "inserção de CVC de longa permanência para HD" correspondeu a US\$134,56 (DP $\pm 3,65)$, sendo $79,52 \%$ relativos ao custo com material, $16,42 \%$ com MOD da equipe de inserção do CVC, 3,46\% com custo de medicamentos e $0,6 \%$ com custo de soluções. Conclui-se, portanto, que os recursos materiais têm um alto impacto na composição do custeio deste procedimento e fica evidente a imprescindibilidade 
da sua alocação racional, especialmente nos HPEP, que possuem recursos orçamentários limitados.

Como contribuição para a prática profissional, o presente estudo conferiu visibilidade aos aspectos financeiros relativos à "inserção de CVC de longa permanência para HD", procedimento empregado para o manejo das complicações associadas ao AV para HDC. Apesar de buscas recorrentes à literatura, nacional e internacional, não foram encontrados estudos semelhantes que viabilizassem a verticalização da discussão sobre o conhecimento econômico produzido. A lacuna de produções dessa natureza indica a necessidade da realização de pesquisas similares em outros HPEP e em hospitais privados.

\section{REFERÊNCIAS}

1. Leite DS, Camargo NLB de, Cordeiro F de B, Schuinski A de FM, Baroni G. Implications of the use of vascular CDL in hemodialysis patients: analysis of echographic insertion sites. J Bras Nefrol. [Internet]. 2014 [acesso em 19 fev 2019]; 36(3). Disponível em: http://dx.doi.org/10.5935/0101-2800.20140046.

2. Ferguson TW, Tangri N, Rigatto C, Komenda P. Cost-effective treatment modalities for reducing morbidity associated with chronic kidney disease. Expert Rev Pharmacoecon Outcomes Res. [Internet]. 2015 [acesso em 19 fev 2019]; 15(2). Disponível em: http://www.ncbi.nlm.nih.gov/pubmed/25661187.

3. Thomé FS, Veronese JV, Manfro RC. Métodos dialíticos na insuficiência renal aguda. In: Barros E, Manfro RC, Thomé FS, Gonçalves LFS, editores. Nefrologia: rotinas, diagnóstico e tratamento. Porto Alegre: Artes Médicas; 2006 p. 365-80.

4. Rubin R. Dialysis treatment in USA: High costs, high death rates. [acesso em 19 fev 2019]. Disponível em: http://usatoday30.usatoday.com/news/health/2009-08-23-dialysis N.htm?csp=15.

5. Ministério da Saúde (BR). Agência Nacional de Vigilância Sanitária. Diretoria Colegiada. ResoluçãoRDC $n^{\circ} 11$ de 13 de março de 2014. Dispõe sobre os Requisitos de Boas Práticas de Funcionamento para os Serviços de Diálise e dá outras providências. [acesso em 19 fev 2019]. Disponível em: https://www20. anvisa.gov.br/segurancadopaciente/index.php/legislacao/item/resolucao-da-diretoria-colegiada-rdc-n-11de-13-de-marco-de-2014.

6. Ministério da Saúde (BR). Agência Nacional de Vigilância Sanitária. Diretoria Colegiada. Resolução-RDC n. 154 de 15 de junho de 2004. Estabelece o Regulamento Técnico para o funcionamento dos Serviços de Diálise. [Internet]. Brasília; 2004 [acesso em 19 fev 2019]. Disponível em: http://www.saude.mt.gov.br/ upload/controle-infeccoes/pasta9/resolucao rdc n154 2004 regulamento servicos dialise.pdf.

7. Lima AFC, Fuzii SMO, Pinho N de A, Melo ACT, Hashimoto THF. Processo de Enfermagem na prática de hemodiálise: a experiência das enfermeiras de um Hospital Universitário. Rev Enf Ref [Internet]. 2010 [acesso em 23 mar 2019]; II(12). Disponível em: http://www.index-f.com/referencia/2010pdf/12-3945.pdf.

8. Romão Junior JE. Tratamento de Substituição da Insuficiência Renal Crônica. In: Lopes AC (organizador). Tratado de Clínica Médica, volume 2. $2^{\text {a }}$ ed. São Paulo: Roca; 2009. p. 2844-54.

9. Neves Junior MA das, Petnys A, Melo RC, Rabboni E. Acesso vascular para hemodiálise: o que há de novo? J Vasc Bras. [Internet]. 2013 [acesso em 23 mar 2019]; 12(3). Disponível em: http://dx.doi. org/10.1590/jvb.2013.044.

10. Lima AFC. Direct cost of monitoring conventional hemodialysis conducted by nursing professionals. Rev Bras Enferm [Internet]. 2017 [acesso em 23 mar 2019];70(2):357-63. Disponível em: http://dx.doi. org/10.1590/0034-7167-2016-0447.

11. Martins E. Contabilidade de custos. $11^{\text {a }}$ ed. São Paulo: Atlas; 2018. 715p. 
12. Castilho V, Lima AFC, Fugulin FMT. Gerenciamento de custos nos serviços de enfermagem. In: Kurcgant $P$, coordenadora. Gerenciamento em enfermagem. 3 ed. Rio de Janeiro: Guanabara Koogan; 2016. p.170-183.

13. MacRae JM, Oliver M, Clark E, Dipchand C, Hiremath S, Kappel J, et al. Arteriovenous vascular access selection and evaluation. Can J kidney Heal Dis. [Internet]. 2016 [acesso em 23 mar 2019]; 3. Disponível em: http://www.ncbi.nlm.nih.gov/pubmed/28270917.

14. Feldman ZM, Liu LB, Abramowitz SD, Faries PL, Marin ML, Schanzer HR, et al. Hemodialysis vascular access: rising costs as a surrogate marker for patency and function of arteriovenous fistulas. Ann Vasc Surg [Internet]. 2017 [acesso em 23 mar 2019]; 38. Disponível em: http://doi.org/10.1016/j.avsg.2016.08.003.

15. Pereira LR, Carvalho MF, Santos JS, Machado GAB, Maia MAC, Andrade RD. Avaliação de procedimentos operacionais padrão implantados em um serviço de saúde. Arq Ciênc Saúde [Internet]. 2017 [acesso em 20 fev 2020]; 24(4). Disponível em: https://doi.org/10.17696/2318-3691.24.4.2017.840.

16. Palmer KS, Brown AD, Evans JM, Marani H, Russell KK, Martin D, et al. Standardising costs or standardising care? Qualitative evaluation of the implementation and impact of a hospital funding reform in Ontario, Canada. Health Research Policy and Systems [Internet]. 2018 [acesso em 20 fev 2020]; 16(74). Disponível em: http://doi.org/10.1186/s12961-018-0353-6.

17. Nogueira DL, Lira GV, Albuquerque IMN, Linhares MSC. Avaliação dos Hospitais de Ensino no Brasil: uma Revisão Sistemática. Rev. bras. educ. med. [Internet]. 2015 [acesso em 15 maio 2019]; 39(1). Disponível em: http://doi.org/10.1590/1981-52712015v39n1e00772014.

18. Abreu RMD de, Stacciarini TSG, Paula GF de, Camargo FC, Garcia LAA, Pereira G de A. Produção científica da equipe de enfermagem de um hospital público de ensino. Revista Família, Ciclos de Vida e Saúde no Contexto Social [Internet]. 2017 [acesso em 20 fev 2020]; 5(3). Disponível em: http://doi. org/10.18554/refacs.v5i3.2136.

19. Oliveira WT de, Rodrigues AVD, Haddad M do CL, Vannuch MTO, Taldivo MA. Concepções de enfermeiros de um hospital universitário público sobre o relatório gerencial de custos. Rev Esc Enferm USP [Internet]. 2012 [acesso em 12 nov 2018]; 46(5). Disponível em: http://dx.doi.org/10.1590/S008062342012000500021.

20. Moraz G, Garcez A da S, Assis EM de, Santos JP dos, Barcellos NT, Kroeff LR. Estudos de custoefetividade em saúde no Brasil: uma revisão sistemática. Ciênc. saúde coletiva [Internet]. 2015 [acesso em 20 fev 2020]; 20(10). Disponível em: https://doi.org/10.1590/1413-812320152010.00962015.

21. Gonçalves MA, Alemão MM. Avaliação econômica em saúde e estudos de custos: uma proposta de alinhamento semântico de conceitos e metodologias. Rev. Med. Minas Gerais [Internet]. 2018 [acesso em 20 fev 2020]; 28(suppl.5). Disponível em: http://doi.org/10.5935/2238-3182.20180136.

22. Vieira FS. Instituto de Pesquisa Econômica Aplicada. Produção de informação de custos para a tomada de decisão no Sistema Único de Saúde: uma questão para a política pública. [Internet]. IPEA; 2017 [acesso em 20 fev 2020]. Disponível em: http://www.ipea.gov.br/portal/images/stories/PDFs/TDs/ td 2314.pdf.

23. Ministério da Saúde (BR). DATASUS. SIGTAP - Sistema de Gerenciamento da Tabela de Procedimentos, Medicamentos e OPM do SUS [Internet]. Ministério da Saúde; 2020 [acesso em $20 \mathrm{fev}$ 2020]. Disponível em: http://sigtap.datasus.gov.br/tabela-unificada/app/sec/procedimento/ exibir/0418010048/04/2020.

24. Ministério da Saúde (BR). DATASUS. Estrutura da Tabela de Procedimentos [Internet]. Ministério da Saúde; 2020 [acesso em 20 fev 2020]. Disponível em: https://wiki.saude.gov.br/sigtap/index.php/ P\%C3\%A1gina principal.

25. Hsiao J, Lima AFC. Custo direto da hemodiálise em unidade de terapia intensiva adulto. Cogitare Enferm [Internet]. 2015 [acesso em 20 fev 2020]; 20(4). Disponível em: https://pdfs.semanticscholar.org/ bbe9/29bd9ed831623f42136216448413734953bd.pdf. 
26. Efstratiadis G, Platsas I, Koukoudis P, Vergoulas G. Interventional nephrology: a new subspecialty of nephrology. Hippokratia [Internet]. 2007 [acesso em 15 maio 2019]; 11(1). Disponível em: https://www. ncbi.nlm.nih.gov/pmc/articles/PMC2464263/.

27. Gouvêa AL, Lima AFC. Direct cost of connecting, maintaining and disconnecting patient-controlled analgesia pump. Rev Esc Enferm. USP [Internet]. 2014 [acesso em 23 mar 2020]; 48(1). Disponível em: https://doi.org/10.1590/S0080-623420140000100013.

28. Homo RFB, Lima AFC. Direct cost of maintenance of totally implanted central venous catheter patency. Rev Latino-Am Enferm [Internet]. 2018 [acesso em 23 mar 2020]; 26. Disponível em: https://doi. org/10.1590/1518-8345.2263.3004.

29. Pires ABM, Lima AFC. Direct cost of peripheral catheterization by nurses. Rev Bras Enferm [Internet]. 2019 [acesso em 23 mar 2020]; 72(1). Disponível em: https://doi.org/10.1590/0034-7167-2018-0250.

30. Almeida LM de, Silva HTH. Equipamento médico-hospitalar: uma gestão na área da saúde. Interdisciplinary Journal of Health Education [Internet]. 2016 [acesso em 20 fev 2020]; 1(1). Disponível em: http://doi.org/10.4322/ijhe2016007.

COMO REFERENCIAR ESTE ARTIGO:

Azevedo BL de, Melo ACT de, Lima AFC. Custo direto da inserção de cateter venoso central para realização de hemodiálise convencional. Cogitare enferm. [Internet]. 2021 [acesso em "colocar data de acesso, dia, mês abreviado e ano"]; 26. Disponível em: http://dx.doi.org/10.5380/ce.v26i0.73651.

*Artigo extraído da dissertação de mestrado "Custos associados ao manejo das complicações de acesso vascular para hemodiálise". Universidade de São Paulo, 2020.

Recebido em: 11/05/2020

Aprovado em: 09/02/2021

Editora associada: Luciana Alcântara Nogueira

Autor Correspondente:

Antônio Fernandes Costa Lima

Universidade de São Paulo - São Paulo, SP, Brasil

E-mail: tonifer@usp.br

Contribuição dos autores:

Contribuições substanciais para a concepção ou desenho do estudo; ou a aquisição, análise ou interpretação de dados do estudo - BLA, ACTM, AFCL

Elaboração e revisão crítica do conteúdo intelectual do estudo - BLA, ACTM, AFCL

Aprovação da versão final do estudo a ser publicado - AFCL

Copyright ( 2021 Este é um artigo em acesso aberto distribuído nos termos da Licença Creative Commons Atribuição, que permite o uso irrestrito, a distribuição e reprodução em qualquer meio desde que o artigo original seja devidamente citado. 